\title{
Interactive comment on "Observation-based
} implementation of ecophysiological processes for a rubber plant functional type in the community land model (CLM4.5-rubber_v1)" by Ashehad A. \section{Ali et al.}

Ashehad A. Ali et al.

ashehad.ali@uni-goettingen.de

Received and published: 28 February 2019

Anonymous Referee \#2

Ali et al. make a manual calibration of the drought-deciduous PFT in CLM to represent rubber trees. The development of PFT parameterisations for such tree crops is important, as they are rarely explicitly represented in large-scale models. The basic concept of the work is fairly solid, but I have reservations around the justification for the calibrated values and the general applicability of the results. 
We thank the reviewer for reviewing our work. We acknowledge that our model developments involved several assumptions and thus had some level of uncertainty associated with it in the first instance. Therefore, we have removed the sections that involved calibrating soil respiration. We place the reviewer's comment as " $\mathrm{C}$ " and provide our response as " $R$ ".

C1: The major limitation is that some important parts of the parameterisation are highly site-specific, which will substantially limit the potential to easily apply this model to sites outside the low nutrient and climate envelope to which it has been parameterised.

R1: We appreciate this comment very much and thank the reviewer for raising his/her concern. Now, we have performed model evaluations at two contrasting climate zones (Thailand and Cambodia sites) and with presumably different management practices, including high soil fertility. Please see the model evaluation section in the manuscript.

C2: Furthermore, many of the parameterisation choices are only loosely supported and may be implicitly incorporating other factors such as climate biases. I would have preferred to see a parameterization which incorporated more flexibility for site conditions - particularly if there are any plans to incorporate this more routinely into a trunk version of CLM where users may be unaware of the caveats of this particular PFT. A clear statement in the conclusions and abstract would help avoid this. I suggest that major revisions are required before publication.

R2: We agree with the reviewer. Data limitation is one challenge that we face for the model development, and thus we acknowledge that there is a considerable level of uncertainty in the present model development. We have made a statement in the conclusion as well as in the abstract, where we state now that we "develop and calibrate a site-specific rubber plant functional type (PFT) for the conditions in Indonesia under low soil fertility". Yes, we would like to put this model into the trunk version of CLM but we will do additional tests first.

Printer-friendly version

Discussion paper

Main comments C3: The model should be run for more sites, either for the calibration 
or evaluation, or ideally both, in order to give confidence in its applicability, even within the relatively narrow window of low nutrients and phenology for which it has been parameterised. Several sites are mentioned in Table 1. Why not also run the model for these sites, rather than the current loose comparison to the Jambi results?

R3: Yes, now we have performed model evaluations at two contrasting climate zones (Cambodia and Thailand) and with different management practices and high soil fertility. Please see the model evaluation section in the manuscript.

C4: The paper as it currently stands does not convincingly demonstrate that the new PFT parameterisation performs better than the basic drought deciduous parameterisation for the Jambi site. This would be easily to solve by adding the results for the unparameterized version to all calibration and evaluation figures.

R4: We have now compared the model simulations using the parameters of the default drought deciduous PFT with the simulations of the rubber PFT in a step-by-step format. These results have been incorporated into the manuscript now. The new rubber PFT performs better for predicting leaf area index, net primary productivity, transpiration, leaf-litterfall, root biomass.

Expanding on the point made in the first paragraph, above, there are three examples of parameterisation which are highly site-specific and limit wider model application:

C5: Parameterising leaf longevity to month (line 185) substantially reduces the wider scale applicability of the model. It is fine if it is only intended to apply the model in the location it has been parameterised for, but it is not appropriate if it is intended to apply the model in other locations or for climate change scenarios. Can the longevity not be linked to climate triggers instead?

R5: We tried considering leaf longevity as a function of climate but we could not get the seasonal dynamics as measured. Thus, we now mention in the manuscript the following: "We acknowledge that currently the seasonal variation in leaf longevity is

Printer-friendly version

Discussion paper 
not a function of climate (e.g. soil water potential, minimum rainfall) and has been developed for a location in Jambi, Indonesia. So we currently suggest adjusting this function to the seasonality of the dry/wet season at other locations."

C6: Similarly, increasing root maintenance respiration to account for increased allocation of carbon to alleviate nutrient limitations constrains the model to only be used in these low-nutrient situations. Surely there are studies which have looked at how allocation or respiration varies with nutrient availability that could be used to develop a more general parameterisation?

R6: We no longer focus on increasing root maintenance respiration and thus have removed this section from the manuscript.

C7: The Q10 tweaking is not very convincing. First off, the justification for the shift in the Q10 is that the rubber plantations used for calibration are 0.5 C hotter than forests. In this case the temperature input to the Q10 could logically be raised by 0.5 C, but this is no justification to change the Q10 itself. Secondly, there are other factors here that could lead to the lower soil carbon. Is litter fall lower for instance, because less productivity goes into growth due to the latex harvest? Or is erosion of soil following clear-cut of the original forest at fault?

R7: We agree that tweaking Q10 is not very convincing and therefore we have removed this section from the manuscript too.

C8: I presume that the climate data used was large-scale, rather than a local weather station. In this case, how can the authors be clear that the differences between the observations and the model are down to the parameterisation of the vegetation and not differences between the real site climate and the large-scale climate dataset? There is a real danger that climate biases from a single site are being parameterised into the vegetation response here.

R8: We have made it clear in the manuscript that local weather climate data was used

Printer-friendly version

Discussion paper
Interactive comment 
from the clear-cut condition at all studied sites.

C9: Please make clear whether the soil respiration measurements are total belowground respiration or pure soil respiration (i.e. using root exclusion cores). When reading how they are compared to the model I sometimes think the former and sometimes the latter. This is important for the justification of the parameterisations made.

R9: Soil respiration is the total belowground respiration, which includes the autotrophic and heterotrophic respiration.

C10: The section on model projection under drought (lines 522-527) is a bit underdeveloped. Even setting aside whether the current parameterisation can be expected to perform well under conditions outside the range for which it was parameterised, this section currently doesn't tell us much. It would be better to elaborate mechanistically on how the different scenarios simulated link to different yield reductions - i.e. make some useful hypotheses. Otherwise, why make all these simulations?

R10: We have removed this section completely from the manuscript.

Other comments C11: Line 193. Please explain a bit better the pools here. It is not immediately clear what a "growth pool" is. This might be a pool of labile carbon to be used for growth, or a pool of structural tissue carbon (e.g. wood). I think you mean the latter, but it is not immediately clear.

R11: We have removed this statement from the manuscript.

C12: Line 228. Why is fcur_st defined as this value? If it is a guess, please say so and explain the logic used.

R11: We have also removed the use of "fcur_st". We have improved the description of tapping in the manuscript (see lines 231-239) and it is more realistic now.

C13: Line 227. Please provide a citation or explanation for tapping starting at six years.

Printer-friendly version

Discussion paper

R13: We have provided the citation now. See lines 284-286 in the manuscript. 
C14: Line 280. Please explain the principle aspects of the model spin-up and climate data used, so that the reader does not have to refer to another paper to get the basic idea of the simulation set-up. R14: We have explained the procedure for spinups in the following lines 291-298; specifically, we make a comment as follows: "We performed a model spin-up in two stages; first a pre-industrial simulation spin-up was carried out, where we used 1900's climate data (1900 to 1940) with pre-industrial CO2 concentration for Jambi from CRUNCEP data (Lawrence et al., 2007). We cycled these 40 years of climate data and ran the model for 1000 years in the accelerated mode. Then we ran the model for 500 years in the normal mode to get to the equilibrium state of ecosystem. Next, we carried out the transient simulation from 1941 to 1997, where the climate data is again extracted from CRUNCEP data and historical CO2 concentrations is used".

C15: Line 228. Did you also transfer below-ground carbon to litter following the clearcut?

R15: Yes, we transferred the fineroot carbon and nitrogen into fast litter pools. We assumed that farmers remove the coarse roots from the forest so that they could plant and thus we did not move them into any litter pools. We have stated these in lines 305-307.

C16: Line 333. Pers. comm. from who? Please also be more precise about how this guess was "educated", explaining the logic used.

R16: We have also removed this statement.

C17: Line 334. "with a depth of the dip not so large in the dry season", is not clear, please rephrase.

R17: We have removed this statement.

Printer-friendly version

C18: Line 337. The change of LAI in the dry season is not in Fig. 2.

Discussion paper

R18: We have removed this statement. 
C19: Lines 337-341. Why even explain this experiment? It has not improved the model behaviour and there is no data to support it anyway.

R19: We would like to keep this experiment in the manuscript because it shows that we considered changing the critical value of soil water potential.

C20: Line 363. Citations or logic needed backing up why these are in the range of plausibility. The comparison to existing ranges in CLM PFTs is not convincing.

R20: We have reshaped the focus of the manuscript and pointed out that the assumptions are likely to have uncertainty associated with it.

C21: Line 490. This logic doesn't hold. There could also be a systematic underestimation of leaf turnover rate.

R21: We disagree with the reviewer here and argue that it can be possible if not all of the rubber trees drop their leaves at the same-time in the dry season.

C22: Lines 554-557. Actually, the effect on the carbon cycle of not getting leaf life span correct is not shown. Please quantify this to support the statement made.

R22: We have removed this statement.

C23: Lines 659-661. This is very tenuously supported by the work here. More confidence in the parameterisation and application of the model at these other sites would be required to support this.

R23: We have also removed this statement.

C24: Figure 7. Both panels can be combined into one for easier comparison and to save space. R24: We have removed this figure too. But we still think that the results of this figure was quite novel, emphasizing the need for a dynamic seasonal component of functional traits in models.

Printer-friendly version

Discussion paper

\section{References}


Lawrence, D. M., Thornton, P. E., Oleson, K. W. and Bonan, G. B.: The Partitioning of Evapotranspiration into Transpiration, Soil Evaporation, and Canopy Evaporation in a GCM: Impacts on Land-Atmosphere Interaction, J. Hydrometeorol., 8(4), 862-880, doi:10.1175/JHM596.1, 2007.

Interactive comment on Geosci. Model Dev. Discuss., https://doi.org/10.5194/gmd-2018-236, 2018. 